\title{
Maneuvering Target Detection Based on JRC System in Gaussian and Non-Gaussian Clutter
}

\author{
Yu Yao, Lenan Wu, and Chenmei Zhang \\ School of Information Science and Engineering, Southeast University, Nanjing 210096, China \\ Correspondence should be addressed to Yu Yao; 1057604987@qq.com
}

Received 11 August 2015; Revised 1 December 2015; Accepted 9 December 2015

Academic Editor: Vladimir Turetsky

Copyright (C) 2015 Yu Yao et al. This is an open access article distributed under the Creative Commons Attribution License, which permits unrestricted use, distribution, and reproduction in any medium, provided the original work is properly cited.

\begin{abstract}
Aimed at the problem of detecting maneuvering targets in the Gaussian and sea clutter environments and based on the established motion state model, this paper proposed a new scheme that uses a joint radar-communication (JRC) system with Kalman filter to accurately detect the target with the generalized likelihood ratio test (GLRT) theory and a constant false alarm rate (CFAR) based threshold. Also, the theoretical threshold and probability function of GLRT target detection based on CFAR were given. Moreover, target detection probability of the new JRC system in Weibull and $K$ distribution clutter is deduced. In addition to theoretical considerations, simulations and measurement results of the new JRC systems demonstrate excellent detection performance for maneuvering targets in the Weibull and $K$ distribution channel.
\end{abstract}

\section{Introduction}

In recent years, maneuvering target detection problem has attracted substantial interest, which is very meaningful for modern radars to improve their detection performance [14]. The complexity of target detection is not only related to the target itself, but also influenced by the electromagnetic environment and clutter background. There are various kinds of weak moving targets: (1) small size target with weak radar returns; (2) low-observable stealth target with low radar cross section (RCS); (3) remote target or target in strong clutter with low signal-to clutter/noise ratio (SCR/SNR); and (4) high speed or highly mobile target with limited available energy. The above four kinds of targets can be summarized as "far-range, low-observable, and highly maneuvering" targets. They share a lot in common that, both in time and in frequency domain, the SCR/SNR is too low to compete with clutter and electronic interference, resulting in poor detection performance $[5,6]$.

The joint radar-communications (JRC) system in the literature [7-9] showed excellent constant false alarm rate (CFAR) target detection performance when performing radar measurements for uniform motion targets. A simple amplitude threshold detector in previous JRC system can demodulate the EBPSK modulated pulse echoes, resulting in a simple receiver structure. The impacting filter $[10,11]$ can be digitally implemented, which is beneficial for chip integration. However, target detection performance of the previous JRC system becomes unsatisfactory for maneuvering target detection in non-Gaussian channel.

As the radar range resolution increases, the background clutter may no longer be modeled accurately as a Gaussian random variable $(\mathrm{RV})$. At the higher range resolution, the radar system receives target-like spikes that give rise to nonGaussian observations. In many cases, it has been found that the spiky clutter returns can be suitably modeled by a multidimensional Gaussian mixture distribution or more specifically a spherically invariant random vector (SIRV) [12]. Such well-known clutter distributions are known as the $K$ and Weibull $[13,14]$. In [15] it was shown that from a reasonable and simple phenomenological model of clutter, the clutter returns from multiple pulses of a given range can be modeled as a SIRV distribution.

In this paper, the generalized likelihood ratio test (GLRT) target detection algorithm based on CFAR is used to detect the presence of target. Aimed at the problem of detecting maneuvering targets with high speed in sea clutter and based on the established motion state model, the JRC system with Kalman filter accurately detects the target with GLRT theory and sets the detection threshold. If the power of echo signal 


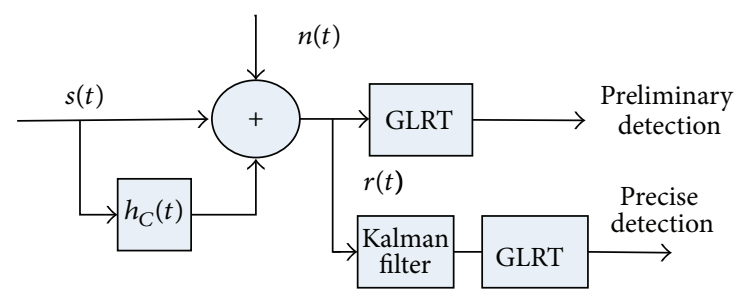

FIGURE 1: The diagram of JRC system model.

after Kalman filtering is greater than the threshold, then the radar system detects the presence of target, otherwise not.

The rest of this paper is organized as follows. Section 2 introduces the model of EBPSK modulated JRC system. Section 3 describes target detection based on GLRT in Gaussian clutter. Section 4 illustrates target detection based on GLRT in Weibull and $K$ distribution clutter via Kalman filtering. In Section 5, the CFAR target detection probability of the JRC system in sea clutter is deduced. Some indicative simulation results and performance analysis are presented in Section 6. And finally, Section 7 gives the conclusion of the paper.

\section{The Model of EBPSK Modulated JRC System}

The EBPSK modulated JRC system model for detection and estimation of maneuvering target in non-Gaussian clutter is shown in Figure 1, where the transmitted signal $s(t)$ is EBPSK modulated signal with random phase encoding, and the echo signal is interfered by additive white Gaussian noise (AWGN) $n(t)$ and Weibull $(K)$ distribution clutter $h_{C}(t)$.

The echo signal would be shown as

$$
r(t)=s\left(t-t_{0}+\frac{2 v}{c_{0}}\left(t-t_{0}\right)\right)\left(1+h_{C}(t)\right)+n(t),
$$

where $t_{0}=2 r_{0} / c_{0}$ is the time corresponding to target initial position, $v$ denotes instantaneous velocity of maneuvering target, and $c_{0}$ represents velocity of light. Motion expression of point target is

$$
\begin{aligned}
& v_{k+1}=v_{k}+a t, \\
& x_{k+1}=x_{k}+v_{k} t+\frac{1}{2} a t^{2},
\end{aligned}
$$

where $v$ represents the target velocity, $x$ describes the target location, and $a$ denotes the target acceleration. For ease of description, assume that $\widehat{a}(t)$ is maneuvering acceleration predicted value of previous time. Random maneuvering acceleration is still a first-order time related process:

$$
\begin{aligned}
& \ddot{x}(t)=\widehat{a}(t)+a(t), \\
& \dot{a}(t)=-\alpha a(t)+w(t),
\end{aligned}
$$

where $a(t)$ represents zero mean colored acceleration noise, $\alpha$ describes the maneuver time constants, and $w(t)$ denotes AWGN. And writing up the expression as an equation

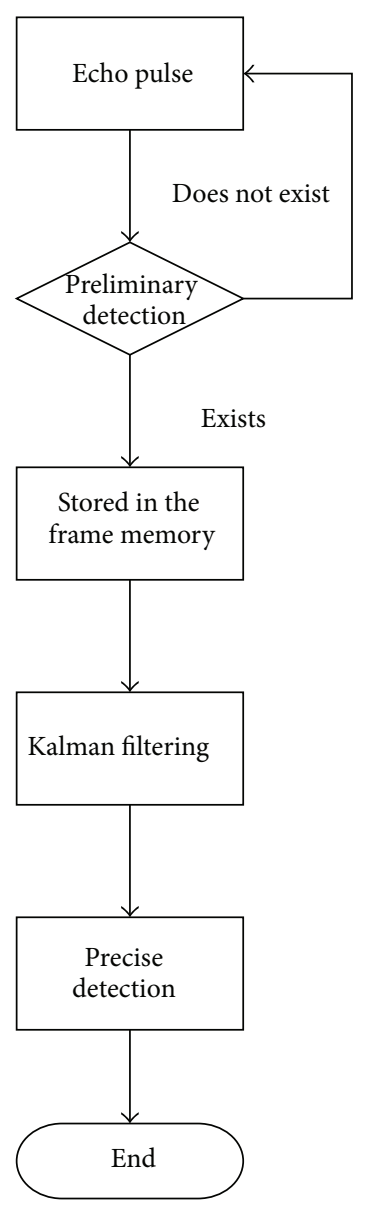

FIGURE 2: The flowchart of signal processing in JRC system.

of motion state, the current statistics adaptive model of maneuvering target would be described as

$$
\begin{aligned}
{\left[\begin{array}{c}
\dot{x}(t) \\
\ddot{x}(t) \\
\dddot{x}(t)
\end{array}\right]=} & {\left[\begin{array}{ccc}
I_{3} & T I_{3} & \frac{T^{2}}{2} I_{3} \\
0 & I_{3} & T I_{3} \\
0 & 0 & I_{3}
\end{array}\right]\left[\begin{array}{c}
x(t) \\
\dot{x}(t) \\
\ddot{x}(t)
\end{array}\right]+\left[\begin{array}{l}
0 \\
0 \\
\alpha
\end{array}\right] \widehat{a}(t) } \\
& +\left[\begin{array}{l}
0 \\
0 \\
1
\end{array}\right] w(t) .
\end{aligned}
$$

For maneuvering target detection, a new scheme with Kalman filter is proposed in the paper, and its flowchart is shown in Figure 2, where echo signal preprocess and target detection in the new JRC system are as follows:

(1) A state model of the target is established, which is in accord with the actual maneuvering situation and convenient for digital processing.

(2) Firstly, the JRC system sends EBPSK modulated pulse with random phase coded.

(3) The JRC system preliminarily detects the target with GLRT theory and sets the detection threshold based 
on CFAR. If the power of echo signal is greater than the threshold, then the radar system detects the presence of target, otherwise not.

(4) When the GLRT detection shows that target is present, based on the location (range), velocity, and acceleration correlation of maneuvering target, a method to process the echo via Kalman filtering based on their phase information correlation is proposed.

(5) Based on the established motion state model, the JRC system with Kalman filter accurately detects the target with GLRT theory and sets the detection threshold based on CFAR. If the power of echo signal after Kalman filtering is greater than the threshold, then the radar system detects the presence of target, otherwise not.

\section{Target Detection Based on GLRT in Gaussian}

CFAR target detection algorithm based on Neyman-Pearson criterion is used as the rule of EBPSK modulated JRC system in this paper. Assume that the target is present $H_{1}$ and absent $H_{0}$, and then the echo signal is

$$
\begin{aligned}
& H_{0}: \mathbf{r}=\mathbf{n}, \quad \mathbf{r} \sim \mathcal{N}\left(0, \mathbf{R}_{0}\right), \\
& H_{1}: \mathbf{r}=\mathbf{S}+\mathbf{n}, \quad \mathbf{r} \sim \mathcal{N}\left(0, \mathbf{R}_{1}\right),
\end{aligned}
$$

where $\mathbf{R}_{1}=\mathbf{R}_{0}+\operatorname{cov}(\mathbf{S})$. Under the condition of $H_{0}$ and $H_{1}$, the distributions of echo signals are also Gaussian distribution, and probability distributions of echo signals would be written as

$$
\begin{aligned}
& p\left(\mathbf{r} ; H_{0}\right)=\frac{1}{\pi^{M}\left|\operatorname{det}\left(\mathbf{R}_{0}\right)\right|} \exp \left(-\mathbf{r}^{H} \mathbf{R}_{0}^{-1} \mathbf{r}\right), \\
& p\left(\mathbf{r} ; H_{1}\right)=\frac{1}{\pi^{M}\left|\operatorname{det}\left(\mathbf{R}_{1}\right)\right|} \exp \left(-\mathbf{r}^{H} \mathbf{R}_{1}^{-1} \mathbf{r}\right) .
\end{aligned}
$$

And the logarithmic likelihood ratio is

$$
\begin{aligned}
l(\mathbf{r}) & =\ln \frac{p\left(\mathbf{r} ; H_{1}\right)}{p\left(\mathbf{r} ; H_{0}\right)}=\ln \frac{\left|\operatorname{det}\left(\mathbf{R}_{0}\right)\right|}{\left|\operatorname{det}\left(\mathbf{R}_{1}\right)\right|}+\mathbf{r}^{H} \mathbf{R}_{0}^{-1} \mathbf{r}-\mathbf{r}^{H} \mathbf{R}_{1}^{-1} \mathbf{r} \\
& =\ln \frac{\left|\operatorname{det}\left(\mathbf{R}_{0}\right)\right|}{\left|\operatorname{det}\left(\mathbf{R}_{1}\right)\right|}+\mathbf{r}^{H}\left(\mathbf{R}_{0}^{-1}-\left(\mathbf{R}_{0}+\sigma_{x}^{2} \mathbf{S} \mathbf{S}^{H}\right)^{-1}\right) \mathbf{r} \\
& =\ln \frac{\left|\operatorname{det}\left(\mathbf{R}_{0}\right)\right|}{\left|\operatorname{det}\left(\mathbf{R}_{1}\right)\right|}+\frac{\left|\mathbf{r}^{H} \mathbf{R}_{0}^{-1} \mathbf{S}\right|^{2}}{1+\sigma_{x}^{2} \mathbf{S R}_{0}^{-1} \mathbf{S}^{H}} .
\end{aligned}
$$

Equation (7) can be simplified, and CFAR target detection algorithm is

$$
T(\mathbf{r})=\left|\mathbf{r}^{H} \mathbf{R}_{0}^{-1} \mathbf{S}\right|^{2} \underset{H_{0}}{\stackrel{H_{1}}{\gtrless}} \gamma .
$$

Set the false alarm probability $P_{\mathrm{fa}}=P\left(T(\mathbf{r})>\gamma \mid H_{0}\right)$. From literature [6], according to Neyman-Pearson lemma, target detection probability would be expressed as

$$
P_{d}=P\left(T(\mathbf{r})>\gamma \mid H_{1}\right)=P_{\mathrm{fa}}^{\sigma_{0}^{2} / \sigma_{1}^{2}},
$$

where $\sigma_{1}^{2}=\sigma_{0}^{2}+\left|\mathbf{S}^{H} \mathbf{R}_{0}^{-1} \mathbf{S}\right|^{2}$ and $\sigma_{0}^{2}=\mathbf{S}^{H} \mathbf{R}_{0}^{-1} \mathbf{S}$. Hence, maximization CFAR target detection probability algorithm is equivalent to

$$
d^{2}=\frac{\left|\mathbf{S}^{H} \mathbf{R}_{0}^{-1} \mathbf{S}\right|^{2}}{\mathbf{S}^{H} \mathbf{R}_{0}^{-1} \mathbf{S}}=\mathbf{S}^{H} \mathbf{R}_{0}^{-1} \mathbf{S} .
$$

From (10), we can get diagonal matrix $\mathbf{R}_{0}=\operatorname{diag}\left(P_{C}\left(f_{-K / 2}\right)+\right.$ $\left.\cdots+P_{C}\left(f_{K / 2-1}\right)\right)$, where $P_{C}(f)$ denotes power spectral density function of clutter.

With the time and velocity correlation of the maneuvering target, the EBPSK modulated JRC system uses Kalman filtering to process echo signals when the GLRT detection shows the presence of target. And the estimation performance can be improved by taking the advantage of prediction and estimation at the same time. The Kalman filtering process can be described as follows:

(1) According to the phase correlation of echo signal, the prediction of echo signal is

$$
\widehat{\mathbf{x}}_{i \mid i-1}=e^{2 a T^{2} / \lambda} \widehat{\mathbf{x}}_{i-1 \mid i-1}
$$

where $\lambda$ denotes the wavelength of echo signal.

(2) According to the prediction results, the MSE matrix of echo signal is

$$
\begin{gathered}
\mathbf{P}_{i \mid i-1}=\boldsymbol{\Phi} \mathbf{P}_{i-1 \mid i-1} \boldsymbol{\Phi}^{H}+\mathbf{Q}, \\
\mathbf{Q}=E\left\{u(n) u^{H}(n)\right\} .
\end{gathered}
$$

(3) Define the Kalman filtering gain matrix as

$$
\begin{aligned}
\mathbf{K}_{i} & =\mathbf{P}_{i \mid i-1} \mathbf{H}^{H}\left(\mathbf{H} \mathbf{P}_{i \mid i-1} \mathbf{H}^{H}+\mathbf{R}_{C}\right)^{-1}, \\
\mathbf{R}_{C} & =E\{\widehat{\mathbf{x}}(n) \widehat{\mathbf{x}}(n)\}, \\
\mathbf{H} & =\mathbf{Q S} .
\end{aligned}
$$

(4) Update echo signal:

$$
\widehat{\mathbf{x}}_{i \mid i}=\widehat{\mathbf{x}}_{i \mid i-1}+\mathbf{K}_{i}\left(\widehat{\mathbf{x}}_{i}-\mathbf{H} \widehat{\mathbf{x}}_{i \mid i-1}\right) .
$$

(5) Update the MSE matrix:

$$
\mathbf{P}_{i \mid i}=\mathbf{P}_{i \mid i-1}-\mathbf{K}_{i} \mathbf{H} \mathbf{P}_{i \mid i-1} .
$$

(6) Run steps (2)-(5) iteratively.

\section{Target Detection in Non-Gaussian Clutter}

We assume that the individual pulses are passed through a spatial whitening filter and thereafter detection takes places, depicted in Figure 3. Hence, the clutter component of the samples in range is statistically decorrelated. The spatial whitening filter essentially removes the correlation from range sample to range sample due to overlapping of the waveform returns from the individual scatterers. 


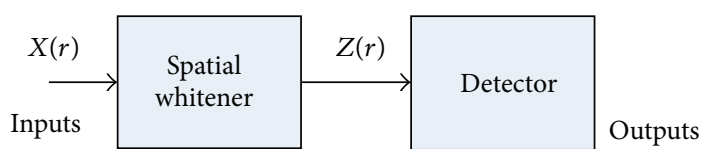

Figure 3: Spatial whitening filtering.

After spatial whitening the texture component on each range sample is an independent $\mathrm{RV}$ with respect to the other range samples. The reason behind spatially whitening the input clutter is that we can conveniently write down its space dependent multidimensional probability density function (PDF). Because all of the RVs are conditionally Gaussian, spatially whitening the clutter allows us to write down the joint space PDF conditioned on the texture RV as a product of the PDFs conditioned on the texture RV at each range.

It is pointed out that matched filtering in range on receiver is not used because the target and clutter have the same power spectrum.

Let the $N$ pulse returns from the moving target be represented by $\left(a(r), e^{j \phi} a(r), e^{j 2 \phi} a(r), \ldots, e^{j(N-1) \phi} a(r)\right)$, where $a(r)$ is the spatially whitened output of the first pulse. Also after this transformation, let $c_{n}(r)$ be the spatially whitened clutter on the $n$th pulse. After the spatial whitening transformation, we desire to devise a detector to distinguish between the two hypotheses:

$$
\begin{aligned}
& H_{1}: \mathbf{z}(r)=a(r) \mathbf{s}_{\phi}+\mathbf{c}(r), \\
& H_{0}: \mathbf{z}(r)=\mathbf{c}(r) .
\end{aligned}
$$

Let $\mathbf{z}_{n}(r)$ be the output of the spatial whitening transformation for the $n$th pulse. Set $\mathbf{z}(r)=$ $\left(z_{1}(r), z_{2}(r), \ldots, z_{N}(r)\right)^{T}, \mathbf{c}(r)=\left(c_{1}(r), c_{2}(r), \ldots, c_{N}(r)\right)^{T}$, and $\mathbf{s}_{\phi}=\left(\begin{array}{lllll}1 & e^{j \phi} & e^{j 2 \phi} & \cdots & e^{j(N-1) \phi}\end{array}\right)^{T}$.

Assuming that $a(r)$ is unknown. The joint PDF of the elements of $\mathbf{z}(r)$ under each hypothesis is given by

$$
\begin{aligned}
& p\left(\mathbf{z}(r) \mid H_{0}\right)=\prod_{\Omega_{r}} \frac{c}{\tau^{N}(r) \operatorname{det}\left(R_{0}\right)} \exp \left[-\frac{1}{\tau(r)} \mathbf{z}^{H}(r)\right. \\
& \left.\cdot R_{0}^{-1} \mathbf{z}(r)\right] \\
& p\left(\mathbf{z}(r) \mid H_{1}\right)=\prod_{\Omega_{r}} \frac{c}{\tau^{N}(r) \operatorname{det}\left(R_{0}\right)} \\
& \quad \times \exp \left[-\frac{1}{\tau(r)}\left(\mathbf{z}^{H}(r)-a(r) \mathbf{s}_{\phi}\right)^{H}\right. \\
& \left.\cdot R_{0}^{-1}\left(\mathbf{z}(r)-a(r) \mathbf{s}_{\phi}\right)\right]
\end{aligned}
$$

where $\operatorname{det}()$ denotes determinant and $c$ is the PDF normalization constant. In this case though, $\tau^{N}(r)(n=1,2, \ldots, N)$ are assumed to be independent Gaussian RVs (random variables) from range cell to range cell. The RVs $\tau^{N}(r)$ are used to model the large power fluctuations associated with the various clutter levels found in different range cells. Standard clutter models of amplitude distribution, such as $K$ and Weibull, are included in the class of Rayleigh mixture distributions which are related to the class of SIRV (spherically invariant random vector) distribution. The maximum likelihood (ML) estimate of $\tau(r)$ under each hypothesis is given by

$$
\begin{aligned}
& H_{0}: \tau_{\mathrm{ML}}(r)=\frac{1}{N} \mathbf{z}^{H}(r) R_{0}^{-1} \mathbf{z}(r), \\
& H_{1}: \tau_{\mathrm{ML}}(r) \\
& \quad=\frac{1}{N}\left(\mathbf{z}(r)-a(r) \mathbf{s}_{\phi}\right)^{H}(r) R_{0}^{-1}\left(\mathbf{z}(r)-a(r) \mathbf{s}_{\phi}\right) .
\end{aligned}
$$

The ML estimate of $a(r)$ under $H_{1}$ is given by

$$
a_{\mathrm{ML}}(r)=\frac{\mathbf{s}_{\phi}^{H} \mathbf{R}_{0}^{-1} \mathbf{z}(r)}{\mathbf{s}_{\phi}^{H} \mathbf{R}_{0}^{-1} \mathbf{s}_{\phi}} .
$$

It can be shown that an equivalent form for the GLRT is

$$
\begin{aligned}
\lambda= & -2(N-1) \\
& \cdot \sum_{\Omega_{r}} \ln \left[1-\frac{\left|\mathbf{s}_{\phi}^{H} \mathbf{R}_{0}^{-1} \mathbf{z}(r)\right|^{2}}{\left(\mathbf{z}^{H}(r) \mathbf{R}_{0}^{-1} \mathbf{z}(r)\right)\left(\mathbf{s}_{\phi}^{H} \mathbf{R}_{0}^{-1} \mathbf{s}_{\phi}\right)}\right] .
\end{aligned}
$$

And the GLRT takes the form

$$
\lambda \stackrel{H_{1}}{\underset{H_{0}}{\gtrless}} T .
$$

Thus, for a chosen false alarm probability the threshold is easily computable.

The JRC system with Kalman filter accurately detects the target with GLRT theory and sets the detection threshold based on CFAR. The processing of Kalman filtering would be described as follows:

(1) According to the prediction results, pulse echo is

$$
\widehat{\mathbf{z}}_{i \mid i-1}=e^{2 a T^{2} / \lambda} \widehat{\mathbf{z}}_{i-1 \mid i-1}
$$

where $\lambda$ denotes the wavelength of echo signal.

(2) According to the prediction results, the MSE matrix of echo signal is

$$
\begin{aligned}
\mathbf{P}_{i \mid i-1} & =\boldsymbol{\Phi} \mathbf{P}_{i-1 \mid i-1} \boldsymbol{\Phi}^{H}+\mathbf{Q}, \\
\mathbf{Q} & =E\left\{u(n) u^{H}(n)\right\} .
\end{aligned}
$$

(3) Define the Kalman filter gain matrix as

$$
\begin{aligned}
\mathbf{K}_{i} & =\mathbf{P}_{i \mid i-1} \mathbf{H}^{H}\left(\mathbf{H} \mathbf{P}_{i \mid i-1} \mathbf{H}^{H}+\mathbf{R}_{C}\right)^{-1}, \\
\mathbf{R}_{C} & =E\{\widehat{\mathbf{z}}(n) \widehat{\mathbf{z}}(n)\}, \\
\mathbf{H} & =\mathbf{Q S} .
\end{aligned}
$$

(4) Update echo signal:

$$
\widehat{\mathbf{z}}_{i \mid i}=\widehat{\mathbf{z}}_{i \mid i-1}+\mathbf{K}_{i}\left(\widehat{\mathbf{z}}_{i}-\mathbf{H} \widehat{\mathbf{z}}_{i \mid i-1}\right) .
$$


(5) Update the MSE matrix

$$
\mathbf{P}_{i \mid i}=\mathbf{P}_{i \mid i-1}-\mathbf{K}_{i} \mathbf{H} \mathbf{P}_{i \mid i-1} .
$$

Run steps (2)-(5) iteratively.

\section{CFAR Detection Probability in Clutter}

Compared to Rayleigh distribution, the envelopes of the PDFs of sea clutter have a longer tail, such as Weibull and $K$ distribution. From literature [16], the PDFs of Weibull and $K$ distribution have two unknown parameters. We discuss presentative OSGO/OSSO-CFAR detector in Weibull and $K$ distribution clutter in this section.

5.1. OSGO/OSSO-CFAR Detection in K Distribution. The cumulative distribution function (CDF) of $K$ distribution would be described as

$$
\begin{aligned}
F(x) & =\int_{0}^{x} f(x) d x=\int_{0}^{x} \frac{2 c}{\Gamma(v)}\left(\frac{c x}{2}\right)^{v} K_{v-1}(c x) d x \\
& =\int_{0}^{c x} \frac{2}{\Gamma(v)}\left(\frac{X}{2}\right)^{v} K_{v-1}(X) d X \\
& =1-\frac{2}{\Gamma(v)}\left(\frac{c x}{2}\right)^{v} K_{v}(c x) .
\end{aligned}
$$

The Ordered Statistic with Greatest Option- (OSGO-) CFAR detector is shown in Figure 4 (the Ordered Statistic with smallest Option- (OSSO-) CFAR has a similar structure). $D$ denotes detecting units, both sides of the $D$ are reference units (also known as reference sliding windows), and both of the lengths of leading and lagging edge reference sliding windows are $M / 2$. Estimation of clutter power level $Z$ can be derived from reference sliding windows. The function sorts $M / 2$ leading and lagging edge reference units based on OSGO-CFAR and selects the maximum value from $X, Y$ as estimation of clutter power level $Z$, respectively (it selects the minimum value based on OSSO-CFAR).

Threshold value $T$ can be calculated according to the corresponding constant false alarm algorithm and set value of false alarm probability. Estimation of clutter power level has self-adaption when the clutter intensity changes.

Figure 4 shows a certain reference window and the signal processing structure for target detection as a flowchart. The first step is to measure the mean clutter power level $Z$. The second step is to multiply this estimation $Z$ by a scaling factor $T$ depending first on the estimation method applied and secondly on the false alarm rate required. The resulting product $T Z$ is directly used as the threshold value. An estimation procedure is proposed which derives the clutter power estimation from the so-called ordered statistic. The two clutter power estimators are then combined into one single value either by further averaging or by maximum selection.

When maneuvering target is absent, background clutters follow $K$ distribution; that is to say, $D, X 1, \ldots, X M$ follow $K$ distribution, and the PDF of the $k$ th sample from the overall in uniform background is expressed as

$$
f_{k}(x)=k\left(\begin{array}{c}
\frac{M}{2} \\
k
\end{array}\right) F^{k-1}(x) f(x)[1-F(x)]^{M / 2-k} .
$$

The CDF of the $k$ th sample is

$$
\begin{aligned}
F_{k}(x) & =\int_{-\infty}^{x} f_{k}(x) d x \\
& =\int_{0}^{x} k\left(\begin{array}{c}
\frac{M}{2} \\
k
\end{array}\right) F^{k-1}(x) f(x)[1-F(x)]^{M / 2-k} d x,
\end{aligned}
$$

where $f(x)$ and $F(x)$ denote PDF and CDF of $K$ distribution, respectively. And for OSGO-CFAR, the PDF of $Z=$ $\max (X, Y)$ is expressed as

$$
f_{z}(z)=f_{X}(z) F_{Y}(z)+f(z) F(z)=\left.2 f_{x}(x) F_{k}(x)\right|_{x=z} .
$$

From (28) and (30), false alarm probability of OSGO-CFAR detector would be described as

$$
\begin{aligned}
P_{\mathrm{fa}} & =P(D \geq T Z)=\int_{0}^{\infty} \int_{T}^{\infty} f(x) f_{z}(z) d x d z \\
& =\int_{0}^{\infty}[1-F(T z)] f_{z}(z) d z \\
& =\int_{0}^{\infty} \frac{2}{\Gamma(v)}\left(\frac{T c z}{2}\right)^{v} K_{v}(T c z) \\
& \cdot c k\left(\frac{M}{2}\right)\left[\frac{2(c z / 2)^{v}}{\Gamma(v)}\right]^{M / 2-k+1} \cdot K_{v-1}(c z) \\
& \cdot K_{v}^{M / 2-k}(c z)\left[1-\frac{2}{\Gamma(v)}\left(\frac{c z}{2}\right)^{v} K_{v}(c z)\right]^{k-1} \\
& \cdot \int_{0}^{t} c k\left(\frac{M}{2}\right)\left[\frac{2(c x / 2)^{v}}{\Gamma(v)}\right]^{M / 2-k+1} K_{v-1}(c x) \\
& \cdot K_{v}^{M / 2-k}(c x) \\
& \cdot\left[1-\frac{2}{\Gamma(v)}\left(\frac{c x}{2}\right)^{v} K_{v}(c x)\right]^{k-1} d x d z,
\end{aligned}
$$

where $T$ denotes threshold factor. From (31), when shape factor $v$ is known, false alarm probability $P_{\mathrm{fa}}$ has nothing to do with scaling factor $c$. Therefore, OSGO-CFAR detector has the constant false alarm performance in $K$ distribution clutter, and so does OSSO-CFAR detector. The relationship between $P_{\mathrm{fa}}$ and threshold coefficients $T$ is taken by numerical integral method.

The optimal values of $k$ need to be calculated in order to optimize performance of detector in clutter. Based on CFAR detection, the aim of selecting value of $k$ is to make detection 


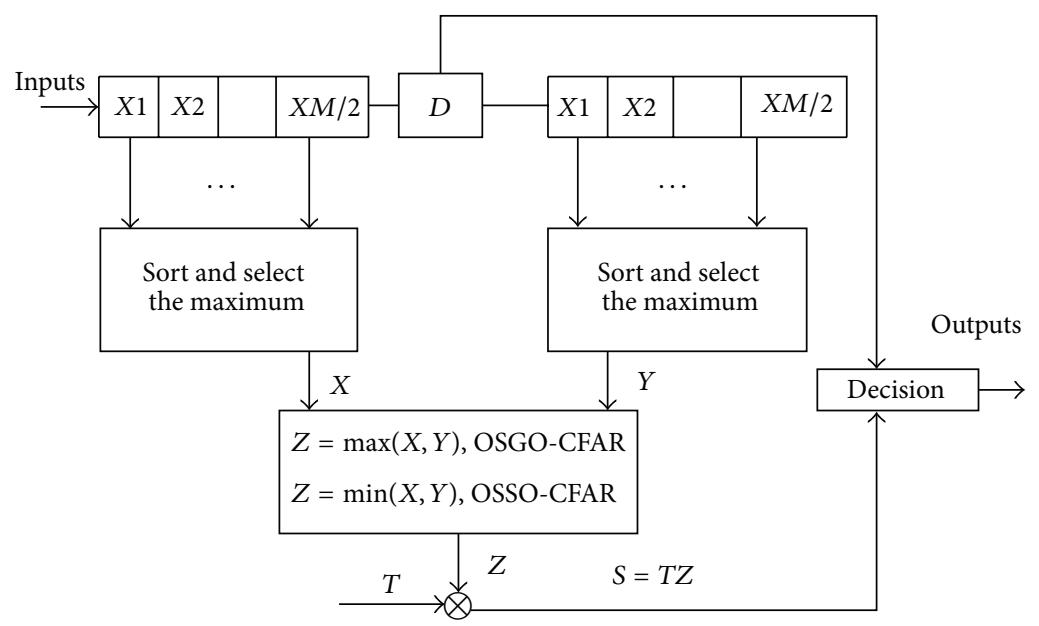

FIGURE 4: OSGO-CFAR detector.

probability $P_{d}$ maximization. We use adaptive detection threshold (ADT) as measurement criteria in this paper. The sum of sinusoidal signal and $K$ distribution calls $K$-Rice distribution and its PDF is

$$
f\left(\frac{x}{H_{1}}\right)=\int_{0}^{\infty} \frac{x}{r} \exp \left[-\frac{x^{2}+A^{2}}{2 r}\right] I_{o}\left(\frac{A x}{r}\right) f(r) d r
$$

where $r$ follows Gamma distribution and $A$ is the magnitude of echo signal. The equation applies to unfluctuation target and Swerling 1 model target. For Swerling 2 model target, $A$ follow Rayleigh distribution. A unified formula of detection probability of CFAR detector is

$$
P_{d}=\int_{0}^{\infty} \int_{T}^{\infty} f\left(\frac{x}{H_{1}}\right) f(z) d x d z
$$

5.2. CFAR Detection in Weibull Distribution. The PDF of random variable $Z=X_{k}$ is

$$
\begin{aligned}
f_{k}(z)= & k\left(\begin{array}{c}
N \\
k
\end{array}\right) \frac{c}{B}\left(\frac{z}{B}\right)^{c-1}\left[\exp \left[-\left(\frac{z}{B}\right)^{c}\right]\right]^{N-k+1} \\
\cdot & {\left[1-\exp \left(-\frac{z}{B}\right)^{c}\right]^{k-1}, \quad z \geq 0 }
\end{aligned}
$$

where $k \in\{1,2, \ldots, M\}$ and $B$ and $c$ denote scale and shape parameter, respectively. Shape parameters $1.160 \leq C \leq 1.783$,

$$
\begin{aligned}
P_{\mathrm{fa}} & =\int_{0}^{\infty}\left[1-F_{0}(T z)\right] f_{k}(z) d z \\
& =k\left(\begin{array}{c}
M \\
k
\end{array}\right) \int_{0}^{\infty} \frac{c}{B}\left(\frac{z}{B}\right)^{c-1}\left[\exp \left[\left(\frac{z}{B}\right)^{c}\right]\right]^{N-k+1+T^{c}} \\
& \cdot\left[1-\exp \left(-\frac{z}{B}\right)^{c}\right]^{k-1} d z .
\end{aligned}
$$

From (35), the value of factor $T$ has nothing to do with average interference power $B$ of Weibull distribution sample. So it is a CFAR method, and false alarm probability would be simplified as

$$
P_{\mathrm{fa}}=k\left(\begin{array}{c}
M \\
k
\end{array}\right) \frac{\Gamma\left(N-k+1+T^{C}\right) \Gamma(k)}{\Gamma\left(N+1+T^{C}\right)} .
$$

Through the constant false alarm processing, the decision threshold $S$ (factor $T$ ) would be calculated for a given false alarm probability $P_{\mathrm{fa}}$, the length of reference units $M$ and $k$.

Set false alarm probability $P_{\mathrm{fa}}=1 \times 10^{-6}, K$ distribution clutter average power $\mu=2$, and the length of reference units $M=32$. Shape parameters $v=1.5$ and 5. From [10], when $k=3 / 4 M \sim 7 / 8 M$, target detection performance of OSSOCFAR detector is optimal. When $k=3 / 8 M \sim 7 / 16 M$, target detection performance of OSGO-CFAR detector is optimal. For multiple target detection, the detection capability of OSSO/OSGO-CFAR detector would get stronger as the value of $k$ gets smaller.

\section{Simulation Results}

In this section, the performance of the JRC system with Kalman filtering is simulated. In Table 1, a summary of the most important parameters of the simulation model is provided.

Simulated Orbit 1. At the beginning, the target bears a constant velocity, and its initial value is $450 \mathrm{~m} / \mathrm{s}$. The target is moving in uniformly accelerated motion between 51st and 100th scanning intervals, and accelerated velocity is $40 \mathrm{~m} / \mathrm{s}^{2}$. And the target is moving in uniform motion again between 101st and 150th scanning intervals.

Simulated Orbit 2. At first, the target is moving in constant velocity along the $x$-axis, the initial velocity is $450 \mathrm{~m} / \mathrm{s}$, the target is moving in circular accelerated motion from the 51st scan cycle, and centripetal acceleration is $20 \mathrm{~m} / \mathrm{s}^{2}$. And the target is moving in uniform motion again after the end of a period. 
TABLE 1: Simulation parameters.

Parameter value

Carrier frequency: $f_{1}=10 \mathrm{GHz}$

Intermediate frequency: $f=100 \mathrm{MHz}$

Sample frequency: $f_{s}=10 \mathrm{GHz}$

EBPSK modulation parameters: $N=7 K=2$

False alarm probability: $P_{\mathrm{fa}}=10^{-6}$

Target detection probability: $P_{d}=0.9$

The number of pulses: $N=10$

Pulse width: $T=200 \mu \mathrm{s}$

Simulated Orbit 1:

Initial velocity: $450 \mathrm{~m} / \mathrm{s}$; accelerated velocity: $40 \mathrm{~m} / \mathrm{s}^{2}$

Simulated Orbit 2:

Initial velocity: $450 \mathrm{~m} / \mathrm{s}$; centripetal acceleration: $20 \mathrm{~m} / \mathrm{s}^{2}$

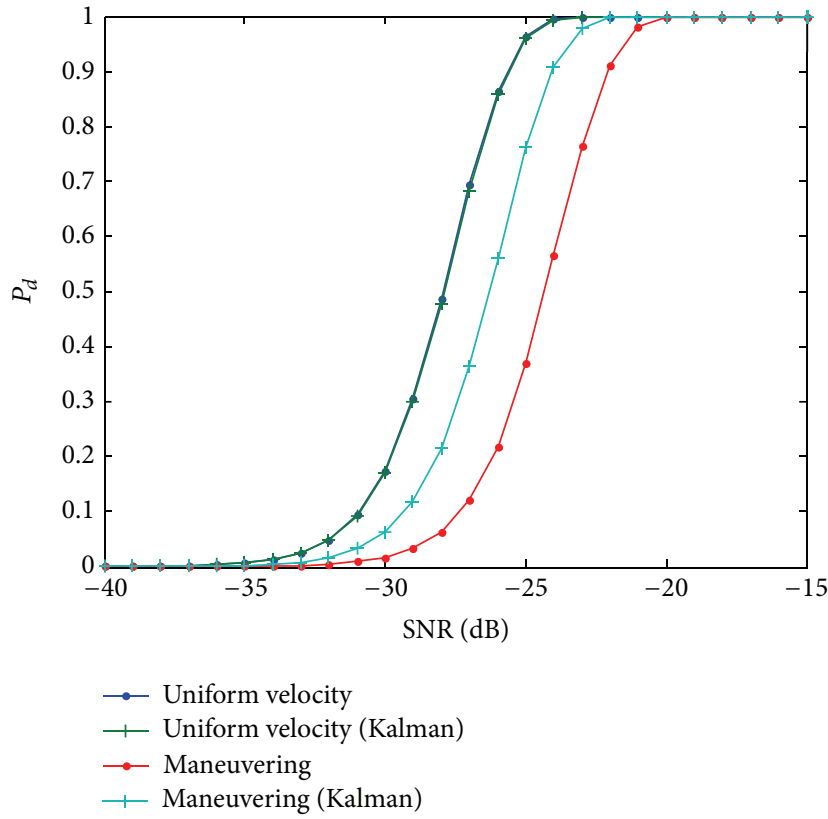

FIGURE 5: Simulated Orbit 1 target detection.

In the following simulations, the relations between CFAR target detection probabilities and the SNR are shown in Figures 5-8. The results are obtained from 4000 Monte Carlo simulations under different SNRs.

Simulation 1. Simulated Orbit 1 target detection is shown in Figure 5.

Figure 5 shows Simulated Orbit 1 target detection probability comparisons for EBPSK modulated pulse echo signal with or without Kalman filtering at the receiver.

For Simulated Orbit 1 maneuvering target, when the detection probability $P_{d}$ is $90 \%$, the SNR performance of the JRC system with Kalman filtering would be improved by approximately $2.4 \mathrm{~dB}$ as compared with the proposed system in AWGN channel.

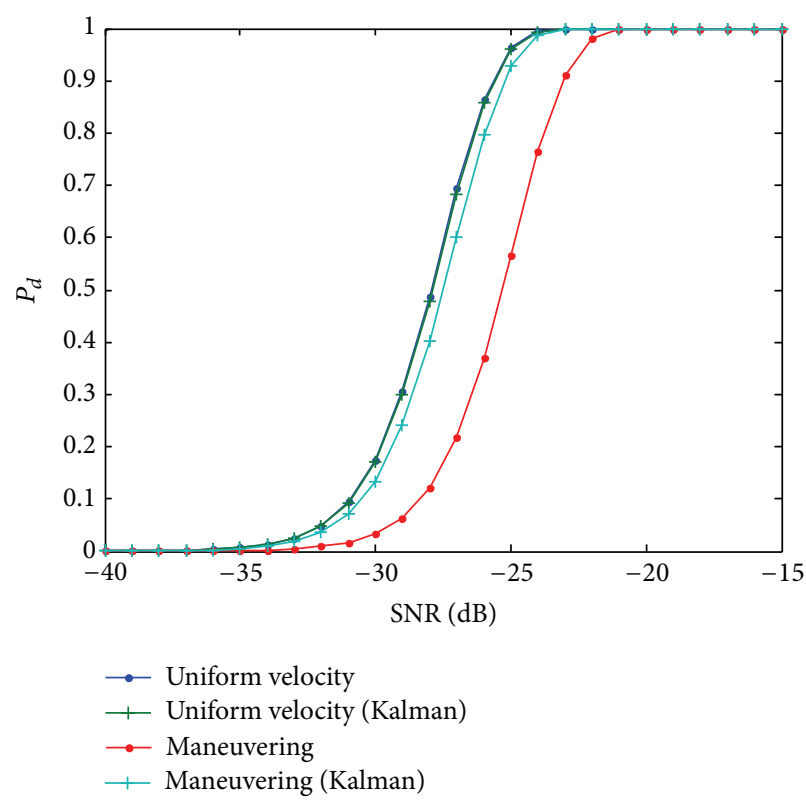

FIgURE 6: Simulated Orbit 2 target detection.

For uniform motion targets, when the detection probability $P_{d}$ is $90 \%$, the SNR performance of JRC system did not improve after Kalman filtering as compared with the proposed system in AWGN channel.

Simulated Orbit 2 target detection simulation is shown in Figure 6.

Figure 6 shows Simulated Orbit 2 target detection probability comparisons for EBPSK modulated pulse echo signal with or without Kalman filtering at the receiver.

For Simulated Orbit 2 maneuvering target, when the detection probability $P_{d}$ is $90 \%$, the SNR performance of the JRC system with Kalman filtering would be improved by approximately $2 \mathrm{~dB}$ as compared with the proposed system in AWGN channel. And detection performance of maneuvering target 2 is better than maneuvering target 1 .

Simulation 2 (Weibull Distribution Clutter). A Weibull distribution clutter model with 32 ranging units is established. Low-power is located between range units 1 and 16. Clutter edger is located in range unit 17. High-power clutter is located between range units 17 and 32 . The length of reference units $M=32$, and shape parameter $c=1.8$. Take $k=3 M / 4$ in the following simulation.

Figure 7 shows CFAR target detection probability comparisons of the JRC systems with or without Kalman filtering in Weibull distribution clutter channel.

For Simulated Orbit 1 maneuvering target, when $P_{d}=$ $90 \%$, the SNR performance of the JRC system with Kalman filtering would be improved by approximately $2.5 \mathrm{~dB}$ as compared with the proposed system.

For uniform motion targets, when $P_{d}=90 \%$, the SNR performance of the JRC system with Kalman filtering would be improved by approximately $0.5 \mathrm{~dB}$ as compared with the proposed system. 


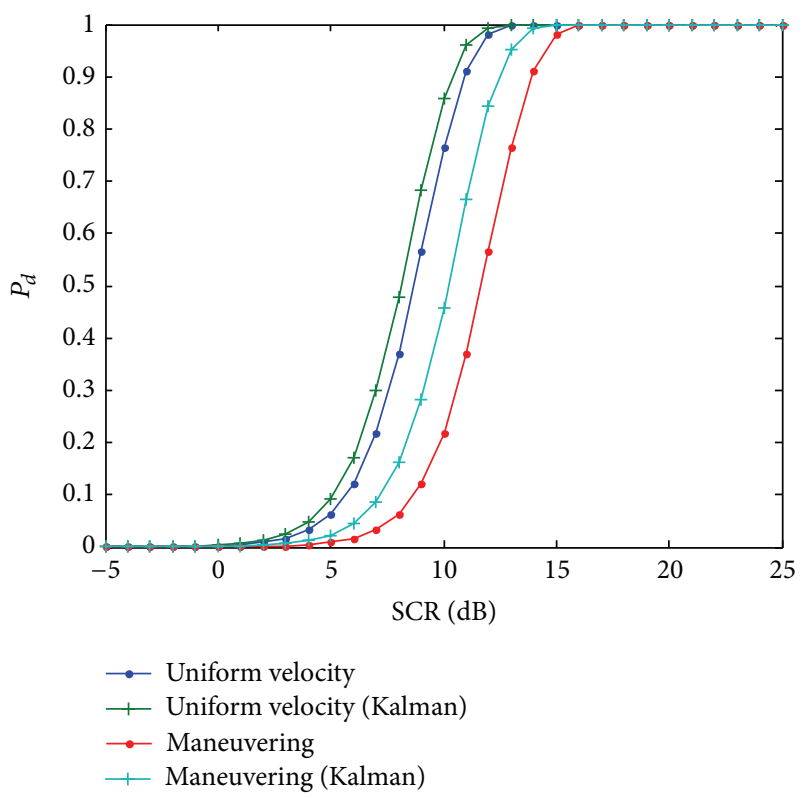

Figure 7: Target detection in Weibull distribution clutter.

Target detection performance of the JRC system in Weibull distribution clutter is much worse than in AWGN.

Simulation 3 (K Distribution Clutter). A $K$ distribution clutter model with 32 ranging units is established. Low-power is located between range units 1 and 16. Clutter edger is located in range unit 17. High-power clutter is located between range units 17 and 32. The length of reference units $M=32$, and shape parameter $c=1.5$. Take $k=3 M / 8$ in the following simulation.

Figure 8 shows CFAR target detection probability comparisons of the JRC systems with or without Kalman filtering in $K$ distribution clutter channel.

For Simulated Orbit 1 maneuvering target, when $P_{d}=$ $90 \%$, the SNR performance of the JRC system with Kalman filtering would be improved by approximately $3 \mathrm{~dB}$ as compared with the proposed system.

For uniform motion targets, when $P_{d}=90 \%$, the SNR performance of the JRC system with Kalman filtering would be improved by approximately $0.6 \mathrm{~dB}$ as compared with the proposed system.

Therefore, target detection performance of the JRC system in $K$ distribution clutter is slightly worse than that in Weibull distribution clutter.

\section{Conclusions}

The detection and estimation problems of maneuvering target are considered, and a new JRC working scheme with Kalman filter is proposed in this paper. The established motion state model of the maneuvering target is given. The generalized likelihood ratio test (GLRT) target detection algorithm based on constant false alarm rate (CFAR) is used to detect the presence of target. When target appears, the Kalman filtering approach is used to estimate EBPSK

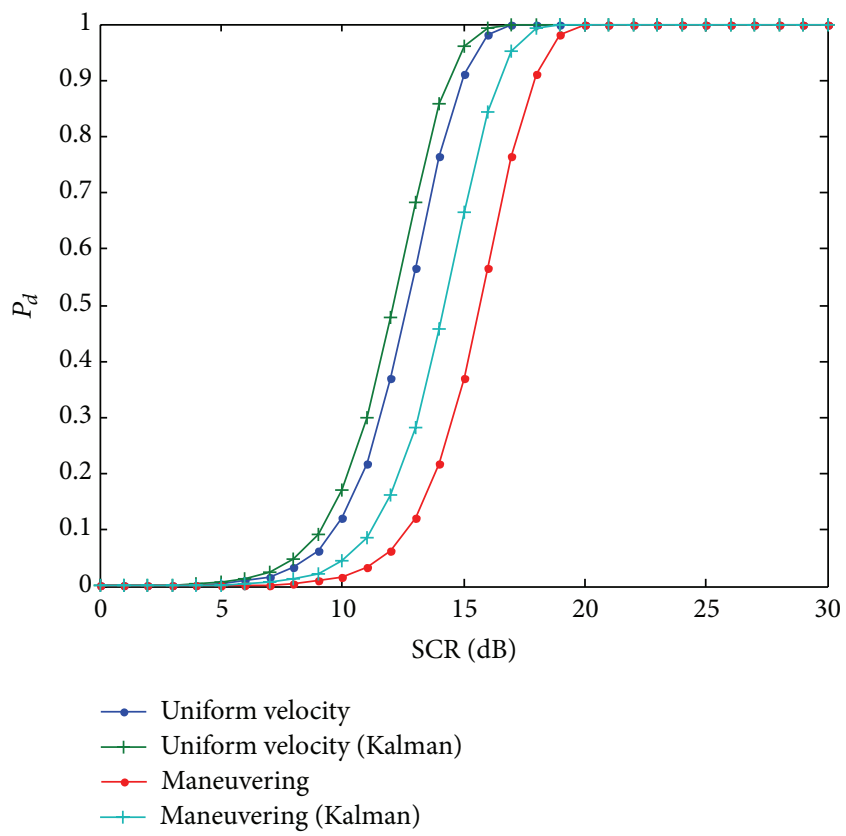

FIgURE 8: Target detection in $K$ distribution clutter.

modulated echo pulse. Furthermore, CFAR target detection probability of the new JRC system in Weibull and $K$ distribution clutter is deduced. The theoretical and simulation results presented confirm the improvement of maneuvering target detection performance in the Gaussian and sea clutter channel via Kalman filtering.

\section{Conflict of Interests}

The authors declare that there is no conflict of interests regarding the publication of this paper.

\section{Acknowledgment}

This work was supported by the National Natural Science Foundation of China (61271204 and 61302096).

\section{References}

[1] D. W. Winters, "Target motion and high range resolution profile generation," IEEE Transactions on Aerospace and Electronic Systems, vol. 48, no. 3, pp. 2140-2153, 2012.

[2] X. Chen, H. Yong, J. Guan, and Y. He, "Sea clutter suppression and moving target detection method based on clutter map cancellation in frft domain," in Proceedings of the 6th International Conference on Radar (RADAR '11), pp. 438-441, IEEE, Chengdu, China, October 2011.

[3] M. Xing, J. Su, G. Wang, and Z. Bao, "New parameter estimation and detection algorithm for high speed small target," IEEE Transactions on Aerospace and Electronic Systems, vol. 47, no. 1, pp. 214-224, 2011.

[4] X. Chen, J. Guan, Y. He, and J. Zhang, "Detection of low observable moving target in sea clutter via fractal characteristics 
in fractional Fourier transform domain," IET Radar, Sonar \& Navigation, vol. 7, no. 6, pp. 635-651, 2013.

[5] X. Chen, Y. Chai, F. Cai, and J. Guan, "Application of the sparse decomposition to micromotion target detection embedded in sea clutter," in Proceedings of the International Conference on Radar (Radar '13), pp. 163-166, Adelaide, Australia, September 2013.

[6] X. Chen, J. Guan, Z. Bao, and Y. He, "Detection and extraction of target with micromotion in spiky sea clutter via short-time fractional fourier transform," IEEE Transactions on Geoscience and Remote Sensing, vol. 52, no. 2, pp. 1002-1018, 2014.

[7] C. Sturm and W. Wiesbeck, "Waveform design and signal processing aspects for fusion of wireless communications and radar sensing," Proceedings of the IEEE, vol. 99, no. 7, pp. 12361259, 2011.

[8] Y. Yao and L. Wu, "Performance analysis of single-frequency MPPSK integrated system for ranging applications," The Scientific World Journal, vol. 2014, Article ID 670346, 11 pages, 2014.

[9] Y. Yao and L. Wu, "An EBSPK-/MPPSK-modem based transceiver for radar-communications," International Journal of Aerospace Engineering, vol. 2015, Article ID 732172, 9 pages, 2015.

[10] Y. Yao and L. N. Wu, "Studies of a dual frequency EBSPKMODEM based radar communication transceiver," Journal of Electronics and Information Technology, vol. 36, no. 8, pp. 17861791, 2014.

[11] M. Feng, L. Wu, and P. Gao, "From special analogous crystal filters to digital impacting filters," Digital Signal Processing, vol. 22, no. 4, pp. 690-696, 2012.

[12] J. Sun, W. Fang, and W. Xu, "A quantum-behaved particle swarm optimization with diversity-guided mutation for the design of two-dimensional IIR digital filters," IEEE Transactions on Circuits and Systems II: Express Briefs, vol. 57, no. 2, pp. 141-145, 2010.

[13] T.-J. Ho and B.-S. Chen, "Novel extended Viterbi-based multiple-model algorithms for state estimation of discrete-time systems with Markov jump parameters," IEEE Transactions on Signal Processing, vol. 54, no. 2, pp. 393-404, 2006.

[14] M. Rangaswamy, D. Weiner, and A. Ozturk, "Computer generation of correlated non-Gaussian radar clutter," IEEE Transactions on Aerospace and Electronic Systems, vol. 31, no. 1, pp. 106116, 1995.

[15] K. Gerlach, M. Steiner, and F. C. Lin, "Detection of a spatially distributed target in white noise," IEEE Signal Processing Letters, vol. 4, no. 7, pp. 198-200, 1997.

[16] F. Gini, "Sub-optimum coherent radar detection in a mixture of K-distributed and Gaussian clutter," IEE Proceedings-Radar, Sonar and Navigation, vol. 144, no. 1, pp. 39-48, 1997. 


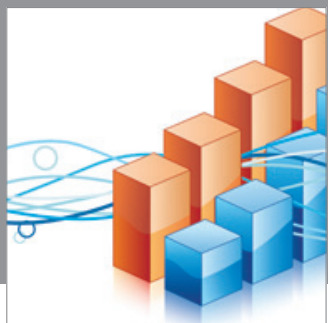

Advances in

Operations Research

mansans

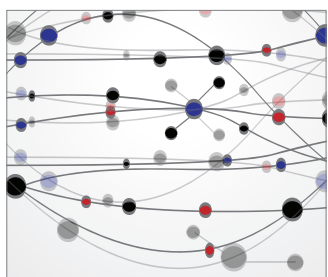

The Scientific World Journal
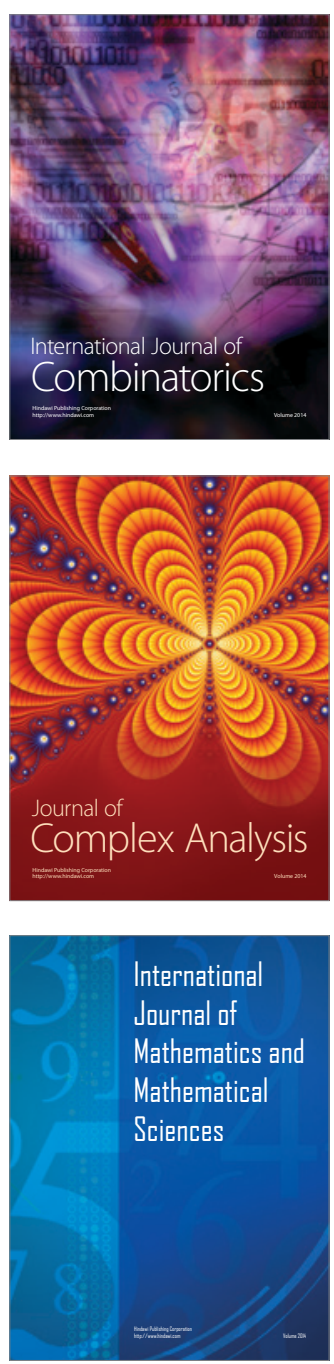
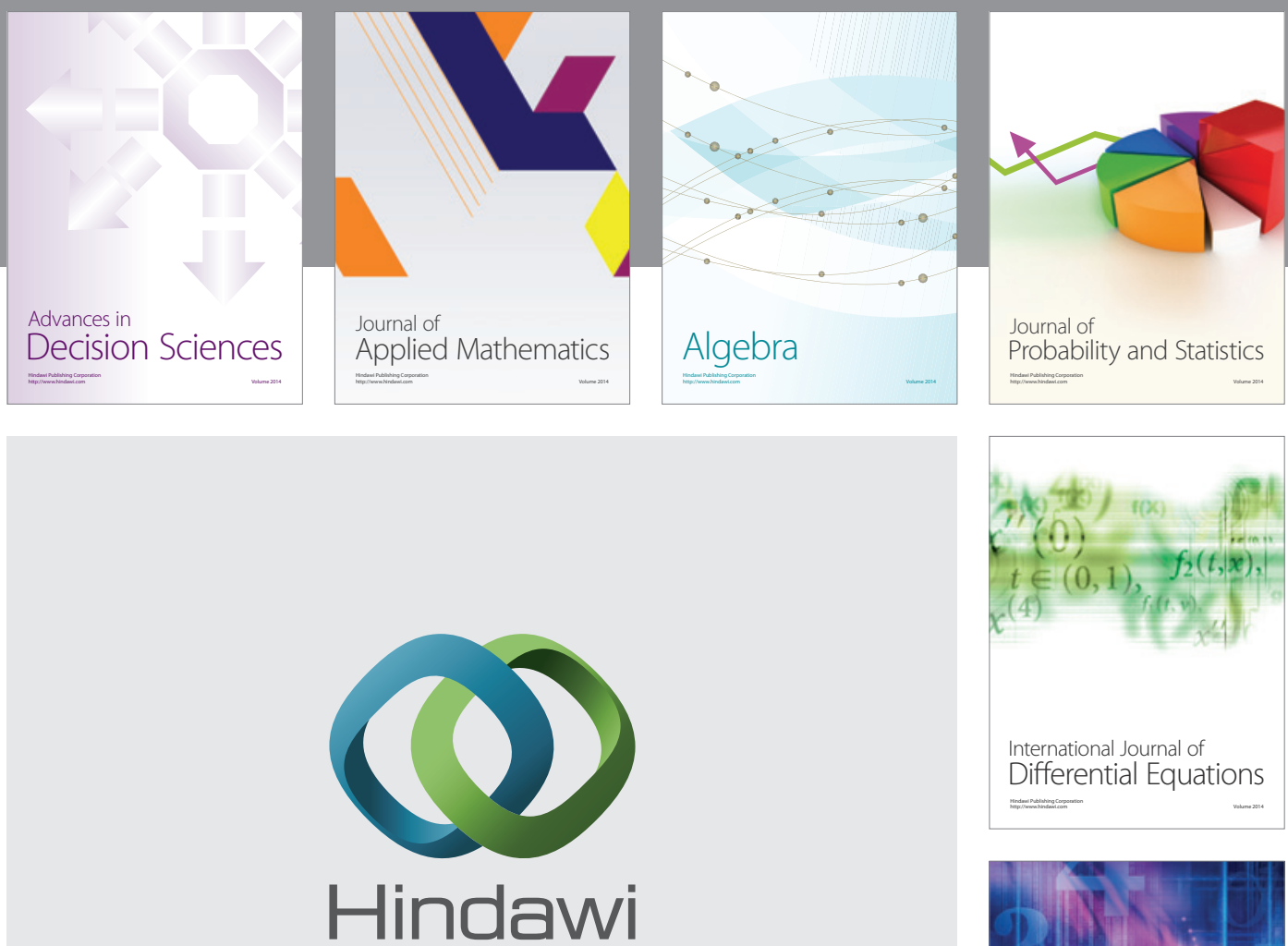

Submit your manuscripts at http://www.hindawi.com
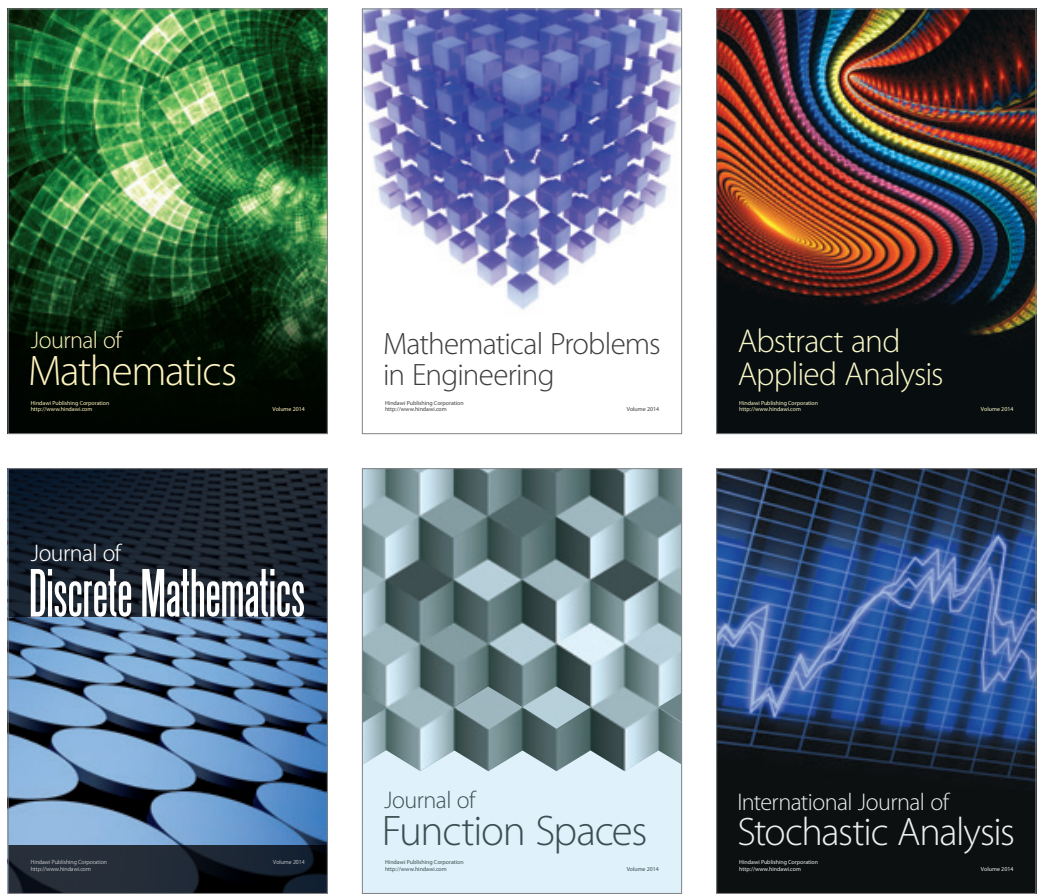

Journal of

Function Spaces

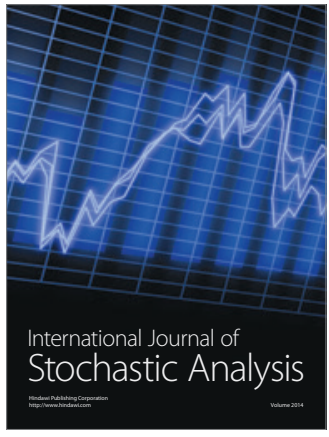

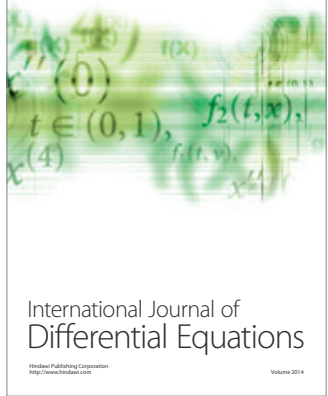
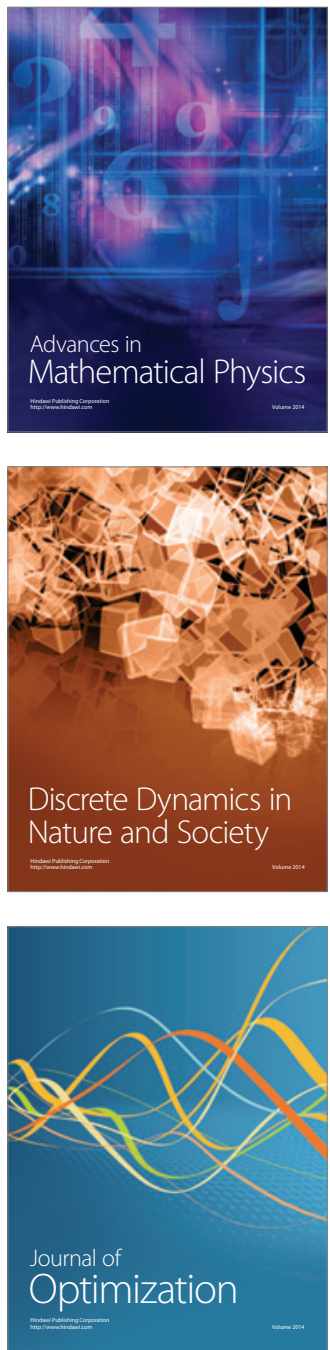